\title{
Postconditioning Does Not Improve Renal Function or Attenuate Tubular Damage in Ischemia/Reperfusion-Induced Acute Kidney Injury in Mice
}

\author{
Shougang Zhuang ${ }^{*}, 1$, Bo Lu² and Maoyin Pang ${ }^{1}$ \\ ${ }^{I}$ Department of Medicine, Brown University School of Medicine, Providence, Rhode Island 02903, USA \\ ${ }^{2}$ Department of Surgery, Medical University of South Carolina, South Carolina 29425, USA
}

\begin{abstract}
Postconditioning (PostC), a series of brief ischemia/reperfusion (I/R) cycles at reperfusion onset, is a recently described approach to attenuate $I / R$ injury in the heart and brain. Here, we examined its effect on acute kidney injury (AKI) induced by ischemia/reperfusion (I/R) injury in a mouse model. C57/black mice were subjected to right nephrectomy and 26-min left renal artery occlusion, and then divided into three groups: Group I, mice were only reperfused for 48 hr; Group II, mice received PostC that was initiated by three cycles of 30-s ischemia with a 30-s interval immediately after initial ischemia before reperfusion; Group III, mice were reperfused for $10 \mathrm{~min}$ after initial ischemia and then received the same regime of PostC prior to reperfusion. At $48 \mathrm{hr}$ after reperfusion, renal function was assessed by measurement of serum creatinine and blood urea nitrogen (BUN), and tubular damage was evaluated by histology. Our results showed that $\mathrm{I} / \mathrm{R}$ injury led to increased serum creatinine and BUN levels and tubular damage. However, PostC did not improve renal function, or attenuate pathological damage to tubules. These results suggest that PostC does not provide a protective effect on renal injury due to ischemia in C57/black mice with these two protocols.
\end{abstract}

Keywords: Postconditioning, ischemia/reperfusion, acute kidney injury, kidney, renal function.

\section{INTRODUCTION}

Ischemic acute kidney Injury (AKI) is a common clinical problem. Although several decades of research have greatly improved our understanding of the mechanisms underlying AKI, effective drugs for treatment of AKI are still not available. Therefore, it is necessary to actively explore other approaches for this disease. Preconditioning, the application of brief, transient periods of nonlethal ischemia before a subsequent lethal episode of ischemia, has been reported to delay the development of renal damage [1-3]. However, this approach is not readily used in clinical practices since renal ischemia in most of cases is only identified when it has already occurred.

In 2003, Zhao et al., introduced the concept of ischemic postconditioning (PostC), which consists of one or more short cycles of reperfusion followed by one or more short cycles of ischemia, immediately after an ischemic phase and before the permanent reperfusion occurs [4]. They showed that the PostC was as efficient as the preconditioning in reducing heart infarct size in a canine model [4]. In the subsequent studies, different observations have been reported regarding the effect of PostC in hearts. While most of studies showed the beneficial effect of this approach on cardiac injury, some of the studies indicated that PostC does not provide protection against heart tissue damage [5-8]. The reasons for those varying effects of PostC remains unclear, but may be related to the duration of ischemia, the algorithm of PostC, animal species, and the gender of animals [5].

*Address correspondence to this author at the Department of Medicine, Brown University School of Medicine, Rhode Island Hospital-Middle House 301, 593 Eddy Street, Providence, RI 02903, USA; Tel: 401-4446847; Fax: 401-444-6849; E-mail: Shougang_Zhuang@brown.edu
The purpose of this study was to assess the effect of PostC on I/R-induced AKI. During the course of the study, other research groups published their results and suggested that PostC was effective in reducing renal damage in rat and murine models of I/R-induced AKI $[1,9,10]$. In contrast to these studies, we revealed that the PostC did not improve the renal function or attenuate the tubular damage in C57/black mice subjected to I/R injury.

\section{MATERIALS AND METHODS}

Experimental Procedures: For this study, a mouse model of right nephrectomy unilateral renal ischemia/rep erfusion was used. Male C57/black mice (approx. 25 g) were anesthetized with pentobarbital and placed on a sterile disposable towel over a warming pad. A midline incision was made, and the renal artery and vein were isolated from surrounding tissue. The right kidney was removed and the left renal arteries and veins were then occluded with a nontraumatic vascular clamp (85grams pressure, Roboz Surg Instruments) for $26 \mathrm{~min}$. This ischemia time was applied as our recent studies showed that serum creatinine and BUN levels were significantly elevated at $24 \mathrm{hr}$ and remained at these levels at $48 \mathrm{hr}$ after ischemic injury [11]. At the end of ischemia, the vascular clamp was removed and subjected to the following treatments: 1) reperfusion for $48 \mathrm{hr}$ (I/R group); 2) three cycles of 30 seconds ischemia with a 30 second interval immediately after initial ischemia before 48 $\mathrm{hr}$ reperfusion (PostC group); 3) 10 min reflow after initial ischemia and then the same regime of PostC before reperfusion (delayed PostC group). In addition, a sham operation was performed for a group of mice in which the renal pedicles were isolated, but no clamp was applied (Fig. 1). There are 6 mice in each group. At the end of experiments, the kid- 
ney was harvested and prepared for histological analysis as described below.

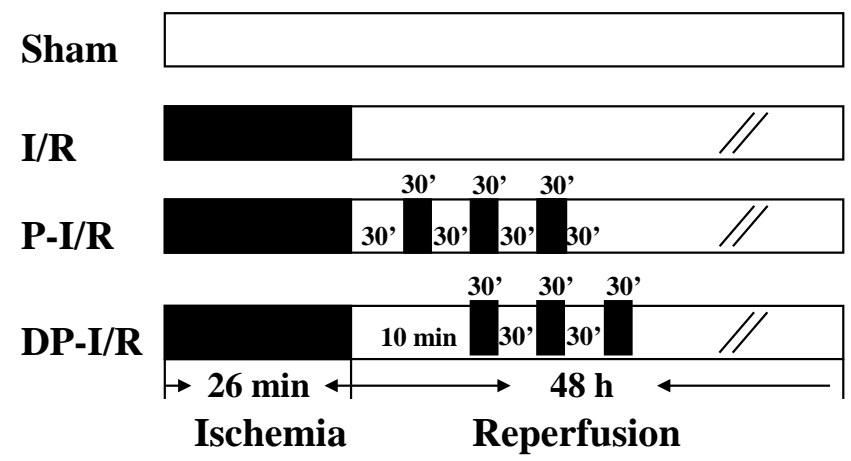

Fig. (1). Experimental algorithm used to determine the effect of PostC on renal function after ischemia and reperfusion. $I / R$, ischemia/reperfusion; P-I/R, ischemia/reperfusion with PostC; DP$\mathrm{I} / \mathrm{R}$, ischemia/reperfusion with delayed PostC.

Histological Examination: Renal tissues were fixed in $4.5 \%$ buffered formalin, dehydrated, and embedded in paraffin. Paraffin sections were deparaffinized and rehydrated before staining for hematoxylin and eosin and examined for tubular injury resulting from $\mathrm{I} / \mathrm{R}$ injury using light microscopy. To assess tubulointerstitial injuries, three representative sections of each kidney ( $n=6$ for each condition) and 10-12 fields/section were examined and scored using semiquantitative indices. Extent of tubular cast formation, tubular dilatation, and tubular degeneration (loss of brush border, detachment of tubular epithelial cells) were scored according to following criteria by two blinded observers: 0 , normal; $1<10 \% ; 2,11$ to $25 \% ; 3,26$ to $50 \% ; 4,51-75 \%, 5>$ 75 of the pertinent area. After scoring, the scores were summed to show the overall tubular damage in the kidney.

Measurement of Creatinine and Blood Urea Nitrogen (BUN): Blood was taken before I/R and at 24 and $48 \mathrm{hr}$ after reperfusion. Serum creatinine and BUN were determined by an automated chemistry analyzer (VITROS 250, OrthoClinical Diagnostics, Rochester, NY, USA) at the MUSC Clinical Laboratory.

Statistical Analysis: All values were expressed as means \pm standard deviation, and one-way analysis of variance (ANOVA) was used with Newman-keuls test for post hoc analysis. In all comparisons, a $p$ value less than 0.05 was considered significant.

\section{RESULTS}

Effect of PostC on Renal Function: Our recent studies showed that mice subjected to $26 \mathrm{~min}$ of renal I/R had significantly increased serum creatinine and BUN levels at 24 $\mathrm{hr}$ and remained at these levels at $48 \mathrm{hr}$ after ischemic injury [11]. To examine the effect of PostC on the renal function in ischemia/reperfusion-induced AKI, we measured serum creatinine and BUN levels at $48 \mathrm{hr}$ after PostC or delayed PostC in this model (see experimental procedures and Fig. (1)). The regimes of PostC were selected based on its protective effects on the heart demonstrated in the dog and rabbit $[4,12,13]$. Delayed PostC was used as a negative control since it has been reported that the protective effect of PostC in the heart was usually observed when it is started immediately after initial ischemia $[5,14]$. Surprisingly, administra- tion of PostC did not significantly reduce the levels of serum creatinine and BUN in mice subjected to I/R injury (Fig. 2). These data suggest that PostC is unable to offer a renoprotective effect in this model.
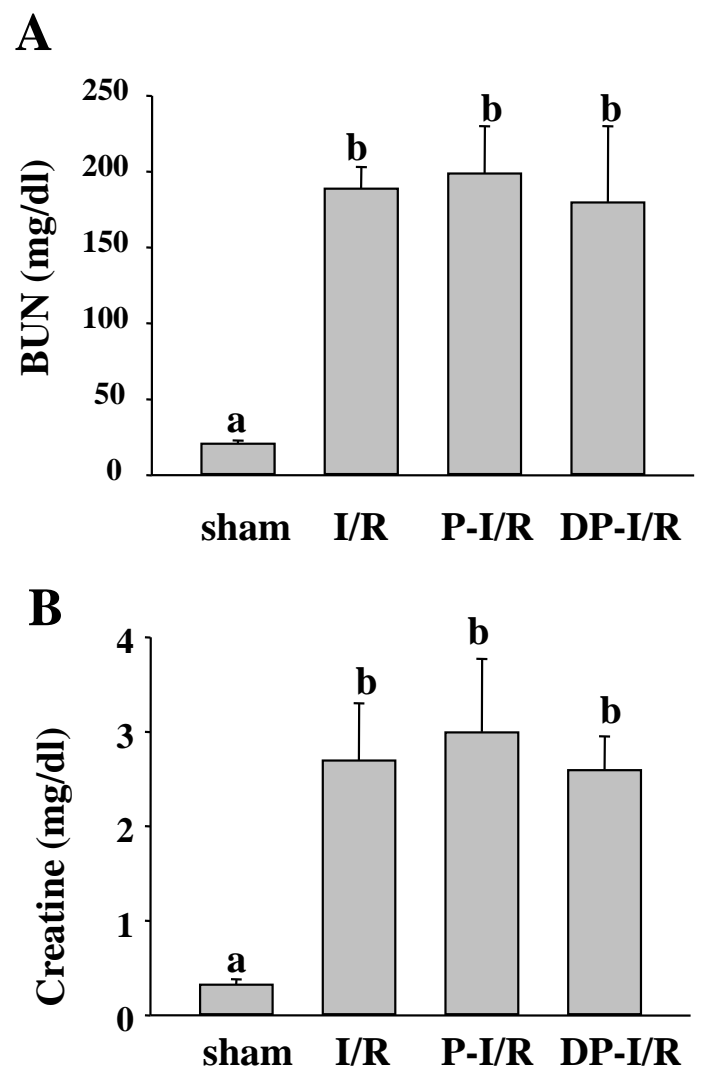

Fig. (2). Effect of PostC on serum creatinine and BUN after ischaemia and reperfusion injury. Mice were subjected to right nephrectomy and 26-min of left kidney occcusion, and then allowed to recovery for $48 \mathrm{hr}$ after treatment with/without PostC as described in the experimental procedures. Data are shown as means \pm SD. Bars with different superscript letters are significantly different from each other $(P<0.05)$.

Effect of PostC on Kidney Morphology After I/R Injury: We also examined the effect of PostC on the kidney morphology. In line with many other observations, ischemic kidney displayed a classic morphology of renal damage, which includes tubular cast formation, tubular dilatation and necrosis, and tubular degeneration (loss of brush border, detachment of tubular epithelial cells). Induction of PostC did not alter these pathological changes (Fig. 3A). Furthermore, scoring of kidney sections for histopathologic damage to the tubules showed an equal degree of tubular damage in all three groups of mice injured by $\mathrm{I} / \mathrm{R}$ with or without PostC treatment. Therefore, we suggest that PostC did not reduce tissue damage in this model.

\section{DISCUSSION}

It has been reported that PostC provides a renoprotective effect on I/R-induced renal injury in rat and mice $[1,9]$. In contrast to these studies, we failed to demonstrate a protective effect of PostC on ischemic AKI in a mouse model. Consistent with our studies, the ineffectiveness of PostC has also been reported in myocardial I/R injuries. For example, Schwartz et al., showed that 30 -s cycles of repetitive ische- 
mia during reperfusion, does not exert a protective effect on pig hearts subjected to lethal ischemia [6]. Dow et al., demonstrated that PostC does not reduce myocardial infarct size in an in vivo regional ischemia rodent model [7]. Furthermore, Hale et al., reported that PostC failed to reduce the extent of the anatomic no-reflow and necrotic damage in hearts of rabbits after acute ischemia [15].
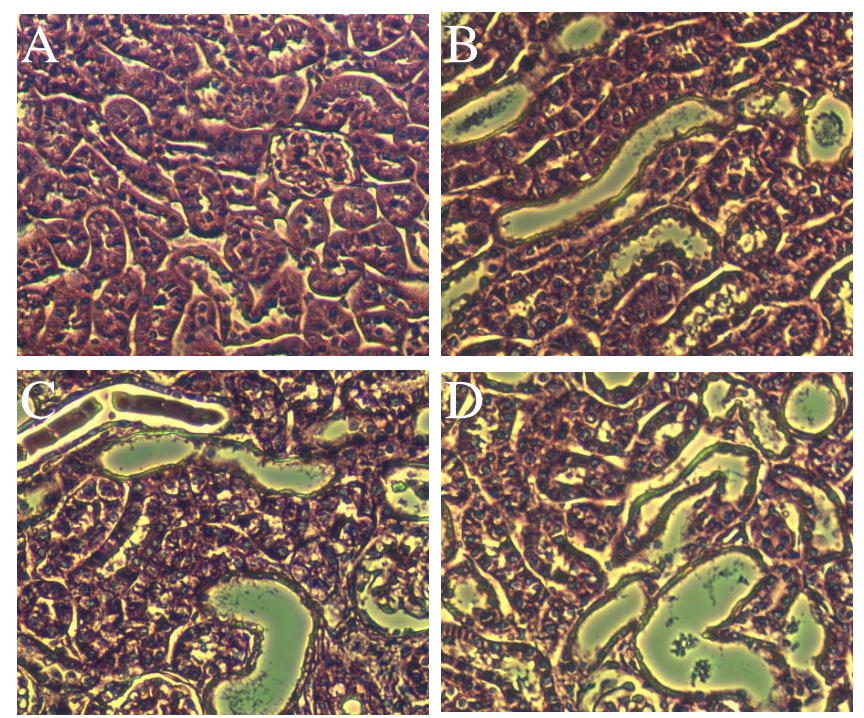

E

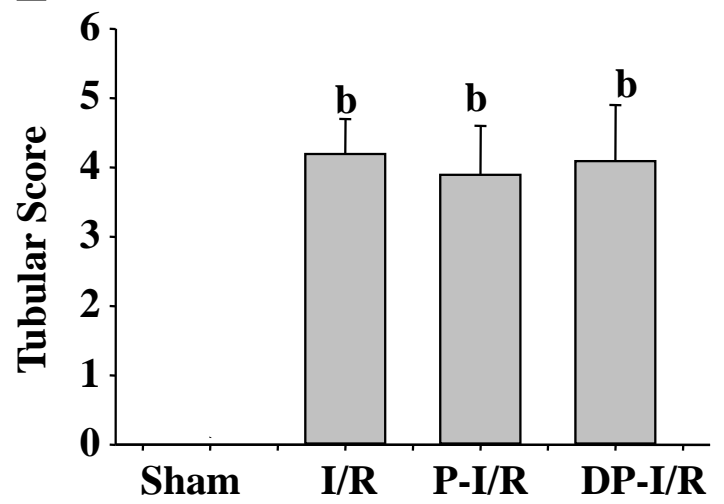

Fig. (3). Effect of PostC on the renal morphology after ischaemia and reperfusion. Mice were subjected to right nephrectomy and 26-min of left kidney occcusion, and then allowed to recovery for $48 \mathrm{hr}$ after treatment with/without PostC as described in the experimental procedures. (A) Sham; (B) I/R plus saline; (C) I/R plus PostC; (D) I/R plus delayed PostC; (E) tabulated data from scoring of renal tissue damage. Data are shown as means \pm SD. Bars with different superscript letters are significantly different from each other $(P<0.05)$. Magnification $=$ X 200 .

The reasons for the discrepancies in the effectiveness of PostC on renal injury between the present study and previous studies remain unknown. In the heart, it has been reported that different PostC algorithms plays an important role in determining the effect of PostC. For example, Chairi and coworkers tested the effect of PostC on I/R-induced cardiac injury using two protocols of PostC in rabbits. While three cycles of 20-s PostC resulted in a significant cardioprotective effect, three cycles of 10-s PostC ischemia failed to reduce infarct size [16]. Schwarz and Lagranha reported that while
PostC obtained with 3 cycles 30 -s ischemia/reperfusion had no beneficial effect in open chest pigs, PostC with intermittent cycles of $1 \mathrm{~min}$ ischemia/reperfusion was effective [6]. However, we used the same regime that was used by Szwarc et al., [9] in that three cycle of 30-s of ischemia and reperfusion, was effective in attenuating renal damage in Swiss mice. Therefore, we suggest that PostC algorithms may not be the sole factor that determines the protective effect of this approach on the kidney.

Recently, Mainintveld et al., have reported that the duration of the preceding period of index ischemia is another key factor in regulating the cardiac effects of PostC [17]. In this study, authors observed that cardioprotection occurred with three cycles of 30-s of ischemia and reperfusion following the $45 \mathrm{~min}$ and $60 \mathrm{~min}$ coronary artery occlusion (CAO), while protection was lost with the longer occlusion duration of 90 and $120 \mathrm{~min}$. Paradoxically, they also observed that with 15-min $\mathrm{CAO}$ as well as 30-min CAO, three cycles of 30 -s of ischemia and reperfusion aggravated irreversible damage [17]. Therefore, PostC can be beneficial, have no effect or be detrimental, depending on the duration of the index ischemia. In the present study, we used $26 \mathrm{~min}$ of ischemia whereas Szwarc et al., [6] used 30-min of ischemia in their study. Although it remains unknown whether our shorter duration of the index ischemia relative to their longer period of ischemia accounts for the inability of PostC to offer a protective effect on renal damage, it will be intriguing to further assess the relationship between the duration of ischemia and the protective effect of PostC in AKI.

In addition, animal gender may also affect the effect of PostC on AKI. Although comparative studies on the effectiveness of PostC have not been conducted in renal ischemia/reperfusion injury in male and female kidneys in the same mouse strain, the effect of PostC on male and female hearts has been examined. In a specifically designed study, Crisostomo et al., showed that while PostC protective effects against ischemia injury was observed in isolated male rat hearts after $25 \mathrm{~min}$ ischemia, the protective effect was absent in female rat hearts exposed to the same time of ischemia [18]. In another study, Penna et al., reported that PostC is less protective against infarct in female than in male rat hearts after 30-min of ischemia [5]. In our current study, we did not observe the renal protective effect of PostC in male mice whereas Szwarc et al., reported that PostC offered a renal protective effect using the same protocol in the female mice with ischemia/reperfusion injury [9]. It is too early to offer an explanation for the differential effect of PostC observed in heart and kidney in terms of gender although different organs may have different responses. PostC warrants further studies to elucidate differences in response of male and female kidneys.

In conclusion, the present study showed that PostC protocols with 30 -s cycles of reperfusion and reocclusion failed to improve renal function and reduce tubular damage in a mouse model of $\mathrm{I} / \mathrm{R}$-induced AKI.

\section{ACKNOWLEDGEMENT}

This work was supported by a grant from National Institutes of Health (DK-071997). 


\section{REFERENCES}

[1] Riera M, Herrero I, Torras $\mathrm{J}$, et al. Ischemic preconditioning improves postischemic acute renal failure. Transplant Proc 1999; 31: 2346-7.

[2] Cochrane J, Williams BT, Banerjee A, et al. Ischemic preconditioning attenuates functional, metabolic, and morphologic injury from ischemic acute renal failure in the rat. Ren Fail 1999; 21: 13545.

[3] Warncke J, David S, Kumpers P, Opherk JP, Haller H, Fliser D. A hibernating kidney - ischemic preconditioning in a renal transplant recipient with a proximal stenosis of the iliac artery. Clin Nephrol 2008; 70: 168-71.

[4] Zhao ZQ, Corvera JS, Halkos ME, et al. Inhibition of myocardial injury by ischemic postconditioning during reperfusion: comparison with ischemic preconditioning. Am J Physiol Heart Circ Physiol 2003; 285: H579-88.

[5] Penna C, Mancardi D, Raimondo S, Geuna S, Pagliaro P. The paradigm of postconditioning to protect the heart. J Cell Mol Med 2008; 12: 435-58

[6] Schwartz LM, Lagranha CJ. Ischemic postconditioning during reperfusion activates Akt and ERK without protecting against lethal myocardial ischemia-reperfusion injury in pigs. Am J Physiol Heart Circ Physiol 2006; 290: H1011-8.

[7] Dow J, Kloner RA. Postconditioning does not reduce myocardial infarct size in an in vivo regional ischemia rodent model. J Cardiovasc Pharmacol Ther 2007; 12: 153-63.

[8] Couvreur N, Lucats L, Tissier R, Bize A, Berdeaux A, Ghaleh B. Differential effects of postconditioning on myocardial stunning and infarction: a study in conscious dogs and anesthetized rabbits. Am J Physiol Heart Circ Physiol 2006; 291: H1345-50.

[9] Szwarc I, Soullier S, Gayrard N, Mejean C, Mourad G, Argiles A. Ischemic postconditioning prevents ischemic acute renal failure. Transplant Proc 2007; 39: 2554-6.
[10] Chen H, Xing B, Liu X, et al. Ischemic postconditioning inhibits apoptosis after renal ischemia/reperfusion injury in rat. Transpl Int 2008; $21: 364-71$

[11] Zhuang S, Lu B, Daubert RA, Chavin KD, Wang L, Schnellmann RG. Suramin promotes recovery from renal ischemia/reperfusion injury in mice. Kidney Int 2009; 75(3): 304-11.

[12] Philipp S, Yang XM, Cui L, Davis AM, Downey JM, Cohen MV. Postconditioning protects rabbit hearts through a protein kinase $\mathrm{C}$ adenosine A2b receptor cascade. Cardiovasc Res 2006; 70: 308-14.

[13] Darling CE, Jiang R, Maynard M, Whittaker P, Vinten-Johansen J, Przyklenk K. Postconditioning via stuttering reperfusion limits myocardial infarct size in rabbit hearts: role of ERK1/2. Am J Physiol Heart Circ Physiol 2005; 289: H1618-26.

[14] Kin H, Zhao ZQ, Sun HY, et al. Postconditioning attenuates myocardial ischemia-reperfusion injury by inhibiting events in the early minutes of reperfusion. Cardiovasc Res 2004; 62: 74-85.

[15] Hale SL, Mehra A, Leeka J, Kloner RA. Postconditioning fails to improve no reflow or alter infarct size in an open-chest rabbit model of myocardial ischemia-reperfusion. Am J Physiol Heart Circ Physiol 2008; 294: H421-5.

[16] Chiari PC, Bienengraeber MW, Pagel PS, Krolikowski JG, Kersten JR, Warltier DC. Isoflurane protects against myocardial infarction during early reperfusion by activation of phosphatidylinositol-3 kinase signal transduction: evidence for anesthetic-induced postconditioning in rabbits. Anesthesiology 2005; 102: 102-9.

[17] Manintveld OC, Te Lintel HM, van den Bos EJ, et al. Cardiac effects of postconditioning depend critically on the duration of index ischemia. Am J Physiol Heart Circ Physiol 2007; 292: H155160 .

[18] Crisostomo PR, Wang M, Wairiuko GM, Terrell AM, Meldrum DR. Postconditioning in females depends on injury severity. J Surg Res 2006; 134: 342-7. 